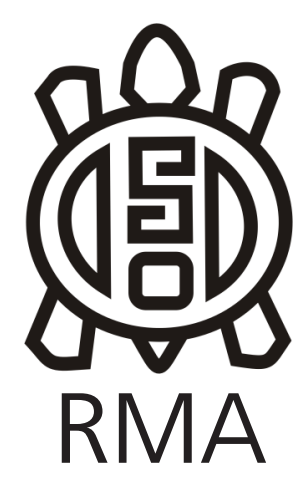

Arqueología

\title{
Reflexiones acerca de la relación entre arqueología, museo y colecciones en Uruguay
}

\author{
Reflections on the relationship between archaeology, museum and \\ collections in Uruguay
}

Gastón Lamas Rivero*, Jimena Blasco* y Eugenia Villarmarzo*

*Laboratorio de Arqueología del Paisaje y Patrimonio (LAPPU), Facultad de Humanidades y Ciencias de la Educación, Unidad asociada al CURE, Universidad de la República, Uruguay. E-mail: gaston.lamas@lappu.edu.uy.

\begin{abstract}
Resumen
El trabajo presenta una reflexión en torno al potencial actual de las colecciones arqueológicas y especialmente del acervo de los museos para las investigaciones arqueológicas. Se parte de un ejemplo concreto como fue el diagnóstico realizado en 2012 por parte del equipo del LAPPU en el Museo Regional de Rocha Milton de los Santos en relación con las investigaciones arqueológicas y patrimoniales en el Área protegida Laguna de Rocha (República Oriental del Uruguay) para a posteriori realizar algunas consideraciones teóricas. El objetivo es brindar reflexiones que puedan a futuro tomarse en cuenta para la definición de acciones de gestión, medidas de conservación y el desarrollo de estrategias de musealización de estas colecciones. Entendemos que mientras se sigan realizando intervenciones arqueológicas y generando colecciones científicas, es necesario, de forma paralela, analizar continuamente desde la propia disciplina el rol que se juega en la construcción del patrimonio y en la conformación de los acervos y discursos dentro de los museos.
\end{abstract}

Palabras clave: Colecciones arqueológicas; Museos; Patrimonio; Conservación; Disociación.

\begin{abstract}
The paper presents a reflection on the current potential of archaeological assemblages and especially the collections of museums for archaeological research. It is based on a concrete example, the diagnosis made in 2012 by the LAPPU team in the Regional Museum of Rocha Milton de los Santos in relation to the archaeological and heritage research in the Protected Area Laguna de Rocha (Oriental Republic of Uruguay) to make some theoretical considerations. The objective is to provide insights that can be taken into account in the future for the definition of management actions, conservation measures and the development of musealization strategies for these collections. We understand that as long as archaeological interventions are continued and scientific collections are created, it is necessary, at the same time, to continuously analyze within archaeology the role they play in the construction of heritage and in the conformation of the collections and discourses within the museums.
\end{abstract}

Keywords: Archaeological Collections; Museums; Heritage; Conservation; Dissociation.

El presente artículo busca discutir y reflexionar sobre el vínculo entre los museos, sus acervos, las colecciones privadas y las investigaciones arqueológicas en el Uruguay. En particular se centra en tratar de definir de qué modo pueden estos acervos (públicos o privados) contribuir a la construcción de conocimiento sobre arqueología y patrimonio. Estos aspectos serán ilustrados a través de un caso concreto: el trabajo con colecciones arqueológicas cuyos materiales provienen de sitios del Paisaje Protegido Laguna de Rocha (en adelante PPLR) y sus alrededores que se encuentran tanto en poder de particulares como en el Museo Regional de Rocha Milton de los Santos (en adelante MRR).
Laguna de Rocha logró su declaración como Paisaje Protegido y su ingreso al Sistema Nacional de Áreas Protegidas (SNAP) en 2010 (ROU 2010). Al inicio de la planificación de los instrumentos de gestión del PPLR se convocó al equipo de arqueología de nuestro laboratorio para comenzar a relevar, identificar e inventariar los sitios arqueológicos existentes en el área (Gianotti \& Villarmarzo 2011). A partir de estos trabajos -y dada la escasa información de partida- se comenzaron a registrar colecciones arqueológicas asociadas al área, tanto privadas (es decir, en manos de particulares y de acceso restringido) como públicas (presentes en museos). Concomitantemente se desarrollaron distintos proyectos 
de extensión e investigación que, entre otras cosas nos permitieron obtener mayor información sobre sitios, colecciones arqueológicas y bienes de interés para los pobladores, los productores y los técnicos del área (Lamas et al. 2013: Villarmarzo 2017).

En ese marco se propuso al MRR, dependiente de la Dirección de Cultura de la Intendencia de Rocha el desarrollo conjunto del proyecto de extensión universitaria «Paisajes culturales y diagnóstico participativo en el Museo Regional de Rocha Milton de los Santos» (Financiado por la Comisión Sectorial de Extensión y Actividades en el Medio, CSEAM de la Universidad de la República, Udelar). Parte de la propuesta estaba dirigida a relevar las colecciones de su acervo e indagar acerca de las posibles asociaciones con el área de estudio. Asimismo, tenía como cometido hacer un diagnóstico general del museo e identificar el potencial de este como lugar para almacenar las colecciones generadas en el marco de investigaciones arqueológicas (Lamas et al. 2012, Villarmarzo 2017) (Figura 1).

Por otro lado, durante el desarrollo de las investigaciones arqueológicas realizadas en el marco del proyecto «Caracterización prehistórica del Paisaje protegido Laguna de Rocha» ${ }^{1}$ se hallaron en el PPLR en paralelo a la identificación de nuevos sitios, otras colecciones privadas que fueron relevadas en 2017 en el marco del proyecto de extensión «Patrimonio en Acción»². Esta última propuesta surgió como punto de partida para actualizar las acciones de colaboración hacia la gestión integral del área. Además, daba respuesta a la preocupación de arqueólogos, guardaparques y tomadores de decisiones (autoridades de gobiernos nacionales y departamentales), por la presencia de prácticas de recolección asistemática y selectiva de materiales arqueológicos de forma intensiva y extendida en el área. Las actividades consistieron en la identificación, registro y diagnóstico de las colecciones. Se realizaron entrevistas semidirigidas para sistematizar información e iniciar un intercambio con los coleccionistas acerca de estas problemáticas, y se elaboraron materiales de sensibilización para trabajar con pobladores, guardaparques y coleccionistas. Además, se comenzó un trabajo de socialización de la experiencia a través de un blog y redes sociales creadas con anterioridad en el marco de otros proyectos de investigación/extensión (Villarmarzo et al. 2017).

\section{Contextualización de la experiencia y metodología}

Las actividades desarrolladas en el MRR son el punto de partida en el presente artículo para tratar de generar un espacio de reflexión acerca del potencial

\footnotetext{
1 Financiado por la Comisión Sectorial de Investigación Científica (CSIC) de la Udelar y desarrollado entre los años 2014 y 2015.

${ }^{2}$ Financiado por la Comisión Sectorial de Extensión y Actividades en el Medio (CSEAM) de la Udelar y desarrollado durante el año 2017.
}

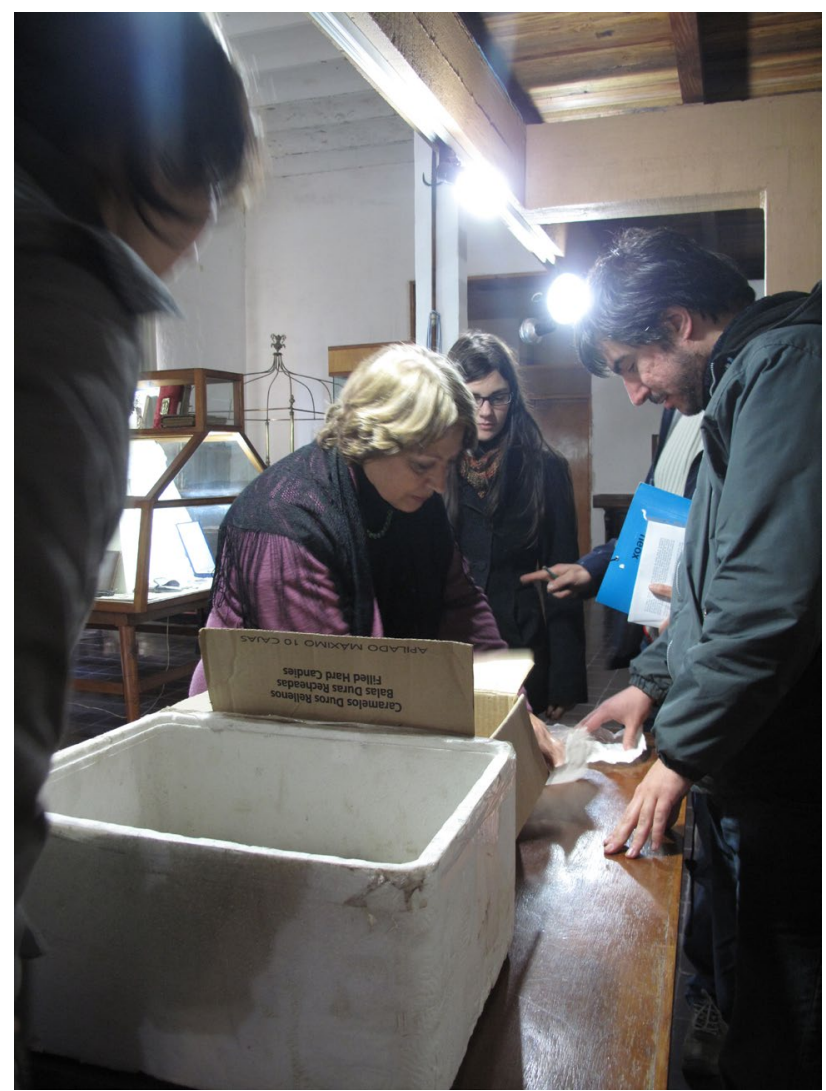

Figura 1. Trabajo en el Museo Regional de Rocha. Año 2012. Fuente: Archivo LAPPU.

Figure 1. Work in progress at de Regional Museum of Rocha. Year 2012. Origin: LAPPU Archive.

de los museos como fuente de información para las investigaciones y lugar de conservación y socialización de las colecciones arqueológicas y su información asociada. Estas se realizaron en el marco del Programa de Educación Patrimonial y Ciencia Pública (en adelante PEPCP) del LAPPU. Dentro de este programa, se propone recurrir al patrimonio cultural como argumento, campo de conocimiento y contexto de acción para co-participar y co-desarrollar procesos de aprendizaje e intercambio (Vienni et al. 2011). Estos conceptos se apoyan entre otros en la perspectiva de la extensión crítica y la integralidad la cual es promovida desde la Universidad de la República (p.e. Arocena, Tommasino et al. 2011). El enfoque de la extensión universitaria en este sentido se sostiene en pilares fundamentales como son los conceptos de diálogo de saberes entre los diferentes actores (universitariosno universitarios, técnicos y comunidades locales), la transformación social, la investigación-acción y la interdisciplina. En el encuentro y aporte de los diferentes saberes de los involucrados en los procesos que se llevan adelante en el territorio es que se produce esta coconstrucción de nuevos conocimientos en este caso en torno al patrimonio.

Las acciones que pusieron en relación a la museología 
con el patrimonio, apuntaron a fortalecer y relacionar los dos pilares básicos del museo: la colección y la sociedad (Valdés Sagüés 2008). En este sentido, el relevamiento y diagnóstico del acervo del museo tenía como objetivo tratar de elaborar propuestas conjuntas entre el equipo universitario y la institución promoviendo la participación de las funcionarias del MRR y equipo técnico que aportaran a la gestión y manejo de las colecciones, su registro e inventario, documentación, conservación, custodia, investigación, seguridad y difusión (Lamas et al. 2012). Para esto se realizó, por un lado, un análisis de la gestión museística a través de un cuadro de FODA (fortalezas, oportunidades, demandas y amenazas) y uno CAME (orientado a aspectos a corregir, afrontar, mantener y explotar) (Tabla 1) y el registro sobre el estado de conservación y documentación de las colecciones arqueológicas y de aspectos generales del museo vinculados a ellas. Asimismo, se realizaron propuestas de productos y/o actividades a llevar a cabo dentro y fuera el museo, co-organizando con el MRR la exhibición de la Exposición Fotográfica "Huellas de la Memoria, una mirada a los Paisajes Culturales de Tacuarembó"3. En el transcurso del proyecto se realizó el relevamiento de la situación de la colección arqueológica expuesta y de aspectos generales del museo vinculados a la misma, utilizando fichas de registro adaptadas para esta actividad. En simultáneo se realizaron entrevistas puntuales a la dirección del museo y constante consulta sobre inventarios y solicitud de acceso a las diferentes áreas. El trabajo de diagnóstico se centró fundamentalmente en la conservación de los bienes arqueológicos si bien otros puntos fueron también tangencialmente abordados. Un aspecto importante tenido en cuenta en el diagnóstico estuvo relacionado al registro y documentación de la colección. Concordamos con Valdés Sagüés en que "Conocer los objetos que el museo posee y su significado, [es la] base indispensable para el desarrollo del resto de las funciones del museo. De ahí la necesidad de desarrollar adecuadamente la documentación de la colección y su investigación" (Valdés Sagüés, 2008:64-65).

Por otro lado, la oferta esencial del museo son las exposiciones (permanentes y temporales) a través de las cuales se pone en práctica una actividad que se torna un instrumento al servicio de la difusión de los contenidos del museo y de sus colecciones, y para difundir a la institución misma (Valdés Sagüés 2008). Pero además, los museos a través de su museografía y de las fórmulas de comunicación que le son específicas, son también instrumentos al servicio del patrimonio cultural que pueden aportar, de forma sencilla y entretenida, a su conocimiento, valoración, conservación, difusión y disfrute (Hernández Hernández 2010). Particularmente los museos arqueológicos permiten poner a disposición

\footnotetext{
${ }^{3}$ Resultado de un proyecto de investigación arqueo-antropológico en distintas localidades del departamento de Tacuarembó. Disponible en http://digital.csic.es/handle/10261/41123. Acceso setiembre de 2017.
}

de todas las personas las interpretaciones sobre el pasado (especialmente aquellas generadas desde la órbita científica) las cuales, aun siendo motivo de disputa, permiten a la sociedad en su conjunto entender mejor el presente y posicionarse sobre él para poder proyectarse a futuro (Hernández Hernández 2010). La autora Francisca Hernández (2010) hace referencia a las disputas sobre las interpretaciones del pasado, donde pueden aparecer diferentes narrativas o memorias que entren en contradicción y en ese sentido no habrá un discurso único lineal y posible para el museo. Es el caso por ejemplo de las distintas "memorias" que pueden aparecer cuando se incorpora la mirada de los pueblos originarios (ver p.e. Salerno y Vigna 2011; Finola 2016).

En esta dirección, como acción concreta se propuso el montaje de la exhibición de la Exposición Fotográfica cuyo objetivo principal fue contribuir a la sensibilización acerca del patrimonio cultural uruguayo, invitando a los visitantes a reflexionar sobre su entorno a través de una mirada hacia los paisajes culturales (Gianotti et al. 2008) en los cuales se difuminan los límites de demarcación que nuestra sociedad impone entre lo lo cultural y lo natural, lo material y lo inmaterial, el presente y el pasado. Tanto para el montaje como para la organización de la charla y la difusión de ambos eventos se trabajó conjuntamente y de forma colaborativa entre el Museo Regional de Rocha y el LAPPU.

\section{Diagnóstico y contexto del Museo Regional de Rocha}

Entendemos al museo como espacio dinámico y al servicio de la sociedad, destinado a la conservación, estudio, comunicación, exposición y educación (Desvallés y Mairesse 2010) que promueve la reflexión en torno al patrimonio y que ocupa un lugar central en el sustento de memorias e identidades (Hernández Hernández 2006). Tanto la definición, como las funciones y el rol de los museos en la sociedad son dinámicos y han-variado sustancialmente a lo largo del tiempo y de acuerdo a la coyuntura específica de cada lugar. Sin embargo, una de las ideas más extendidas en la modernidad ha sido, como señala críticamente Podogorny, la equiparación del museo "a un símbolo de civilización y de estar en el mundo de acuerdo con el tono de los tiempos." (Podgorny 2005:258). Esto está en estrecho vínculo con la capacidad que tienen los objetos de evocación y significación simbólica y que, a través de los procesos de patrimonialización que activan los museos, colaboran a generar discursos sacralizados de versiones ideológicas de la identidad (Prats 2006:72).

En Uruguay, los museos locales y regionales comienzan a formarse a partir de la segunda mitad del siglo $X X$, originándose en base a colecciones privadas o particulares que a través de este proceso se incorporan al ámbito público. En este sentido, el coleccionista adquiere 
protagonismo en la formación de los museos locales y municipales y en la institucionalización acaecida a través del pasaje de las colecciones a la esfera pública, pasando a ocupar en muchos casos cargos de dirección (Malán 2013). Entre otros, la Sociedad de Amigos de la Arqueología (1926-1978) promovió también una visión sobre la recolección de materiales como forma de investigación y preservación que aún perdura. Este proceso deriva entre otras cosas, en una legitimación institucional a la acción de los coleccionistas o aficionados (Pupio 2007) que continuaron con la recolección selectiva y asistemática al amparo muchas veces de las instituciones museísticas, generando una desconexión entre estos saberes y los generados desde la academia.

En cuanto al MRR, fue fundado en 1985 a partir de donaciones del Batallón de Infantería $N^{\circ} 12$ y material arqueológico proveniente de la zona Isla Larga (al norte del Departamento de Rocha). A nivel museográfico presenta una clasificación de su acervo basada en una interpretación de la historia de los pobladores del departamento que sigue un eje temporal de etapas consecutivas, sintetizando y simplificando un proceso complejo de miles de años. En este sentido, la temática principal es la historia regional y local en consonancia con la narrativa clásica sobre el pasado generada desde el Estado Nación, que apela a ancestros fundadores, héroes míticos, batallas e instituciones emblemáticas, entre otros (López Mazz 2004). Concretamente, las unidades temáticas de la exposición permanente del museo son: 1) Paleontología, 2) Los grupos indígenas que habitaron el territorio, con algunas referencias a la Arqueología, 3) Lo rural o gauchesco, 4) La historia de la fundación de Rocha que hace un recorrido histórico desde su etapa prefundacional con referencias a hitos, símbolos y personajes emblemáticos a nivel local y nacional, y 5) El sector de 1900, en el que se exponen ropas típicas y elementos decorativos de época (Figura 2).

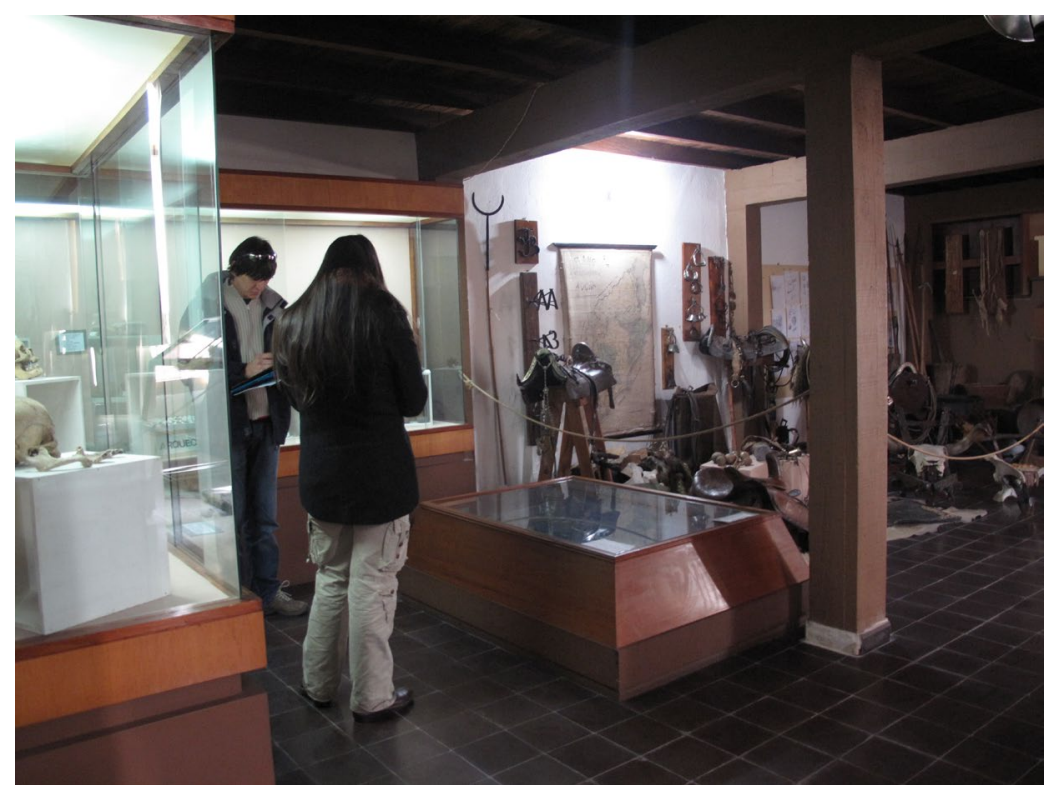

La fundación del MMR, acaecida hacia el final de la dictadura uruguaya, puede asociarse en tiempo y procesos, a las de otros museos con colecciones arqueológicas del país y, concretamente, de Rocha (p.e. Museo Departamental Beltrán Pérez de la ciudad de Castillos). Asimismo, es coincidente con los primeros años de la arqueología profesional en nuestro país. Tras todos estos años, aunque si bien se han propuesto proyectos de musealización in situ y desarrollo de centros de interpretación vinculados a sitios arqueológicos de Rocha (p.e. López Mazz 2008) las décadas de trabajo arqueológico en dicho departamento no se tradujeron en un vínculo fluido entre academia e instituciones museales tradicionales. No obstante, como producto de procesos internos y externos a los museos, en el último lustro, este vínculo se ha fortalecido en nuestro país a través de iniciativas tanto de investigación -con foco en las colecciones en sí mismas como en la relación arqueologíamuseos- como de acciones concretas -relacionadas a la conservación preventiva ${ }^{4}$, documentación y registro, educación y exposición- (p.e. Blasco et al. 2017; Gascue et al. 2015; Sosa et al. 2014). Estos trabajos coinciden con el desarrollo de un proceso teórico-reflexivo sobre la práctica arqueológica y la producción de conocimiento desde dicha disciplina (Cabrera Pérez 2011; Curbelo 2004; López Mazz 2004), el egreso de la primera generación de Técnicos en Museología (Facultad de Humanidades y Ciencias de la Educación, Udelar), la creación en el año 2010 del Proyecto Sistema Nacional de Museos $^{5}$ y la

\footnotetext{
4 Se entiende por Conservación Preventiva "todas aquellas medidas y acciones que tengan como objetivo evitar o minimizar futuros deterioros o pérdidas. Se realizan sobre el contexto o el área circundante al bien, o más frecuentemente un grupo de bienes, sin tener en cuenta su edad o condición. Estas medidas y acciones son indirectas - no interfieren con los materiales y las estructuras de los bienes. No modifican su apariencia" (ICOM 2008).

5 El mismo surge como iniciativa del Ministerio de Educación y Cultura (MEC) con el objetivo de conformar un sistema nacional de fortalecimiento institucional, promoción de la cooperación y
}

Figura 2. Unidad temática sobre indígenas junto a la unidad lo rural y gauchesco dentro del MRR. Año 2012. Fuente: Archivo LAPPU.

Figure 2. Thematic unit on indigenous people next to the rural and gaucho unit within the MRR. Year 2012. Origin: LAPPU file. 
posterior Ley de Museos y Sistema Nacional de Museos de la República Oriental del Uruguay (ROU Ley N¹9037) en 2012, así como a la existencia de convocatorias a proyectos de extensión, culturales y de investigación aplicada en la Udelar y otros organismos públicos (Ministerio de Educación y Cultura, Agencia Nacional de Investigación e Innovación).

\section{Musealización, inventario y conservación}

Se parte de la idea de musealización como apuntan Desvallées y Mairesse (2010):

"...la operación que tiende a extraer, física y conceptualmente, una cosa de su medio natural o cultural de origen para darle un status museal ... La musealización, como proceso científico, abarca el conjunto de actividades del museo: el trabajo de preservación (selección, adquisición, gestión, conservación), de investigación (del que surge la catalogación) y de comunicación (por medio de la exposición, las publicaciones, etc.)". (Desvallées y Mairesse 2010:50-51).

Este proceso científico de musealización, que implica descontextualizar y recontextualizar a los objetos y que involucra a distintas actividades del museo, los objetos y colecciones, requiere de un adecuado registro, investigación e interpretación para que no pierdan sentido (Desvallées y Mairesse 2010). Estos son aspectos que no se dan en muchas de las colectas selectivas de aficionados, ni en el caso de donaciones de particulares (Vienni y Blasco 2014). En contraposición, se da por sentado que el responsable de un proyecto de investigación arqueológico tiene la responsabilidad de velar por el cuidado del patrimonio y publicar los resultados de los análisis e intervenciones (Almansa Sánchez 2011), así como brindar el inventario de piezas.

Hay tres aspectos que son de especial preocupación cuando pensamos en la relación entre colecciones arqueológicas y museos: a) la integridad de la colección vs. la disociación de materiales, b) la necesidad de documentar las colecciones, constituir catálogos y mantener inventarios actualizados, y c) los aspectos de conservación de las colecciones. Teniendo en cuenta esto y a los propósitos de un diagnóstico, interesa conocer la situación en torno a la conservación de los materiales arqueológicos en todas las fases del trabajo arqueológico in situ y en los lugares de depósito y exposición (Vienni 2009).

En el año 2011 fue aprobada Ley de Museos de la República Oriental del Uruguay (ROU Ley N¹9037/11, Artículo 5). Esta plantea para las colecciones museográficas la debida documentación con criterios museológicos de su acervo. Bajo el nuevo panorama de la Ley de Museos los inventarios toman un papel protagónico,

optimización de recursos de los museos uruguayos. donde la protección y conservación del patrimonio de la institución necesariamente estará vinculada al inventario correspondiente. Esta plantea para las colecciones museográficas la debida documentación con criterios museológicos de su acervo. Los inventarios contienen identificación, descripción y localización del objeto. Su fin es la documentación para la divulgación (Llop I Bayó 1996). Es importante tener en consideración que el inventario no evalúa, sólo consigna la existencia de bienes aislados de su contexto, cumpliendo con una función necesaria en el proceso de apropiación. Asimismo, junto a la investigación académica del conjunto de objetos, los inventarios pueden servir de base para la creación de catálogos que refuercen la interpretación y caracterización del acervo, contribuyendo a la valoración del contexto histórico (Vienni 2009).

Al respecto de la conservación y la integridad de una colección, uno de los agentes de deterioro que incide particularmente es la disociación entre diferentes objetos de una colección; entre partes de un mismo objeto y entre el objeto o colección y la información asociada, situación que conduce a su pérdida o mezcla de materiales derivando en la desvalorización de una colección como referencia para el proceso de investigación (Waller y Cato 2009):

"La disociación surge de la tendencia natural de los sistemas ordenados a deshacerse a lo largo del tiempo. Para prevenirla, es necesario modificar los procesos de mantenimiento y otras barreras. La disociación provoca la pérdida de objetos, de su información relacionada o de la capacidad para recuperar o asociar objetos e información." (Waller y Cato 2009:1).

Si los materiales de una colección "están mezclados entre sí, la colección completa se considera comprometida; por consiguiente, unas pocas muestras cruzadas, pueden lograr que una colección pierda buena parte de su valor como referencia y para la investigación" (Waller y Cato 2009:2).

Tanto para la investigación científica como para la labor museográfica es esencial, cotejar que exista un inventario y que la información esté presente. Por otro lado, si además sumamos el deterioro o pérdida física a causa de otros agentes de deterioro relacionados con factores ambientales, humanos y accidentales o por catástrofes (Gómez 2009, Michalsky 2006), no queda más que sentarse a contemplar cómo se borran las huellas del pasado.

En este sentido es posible que a la hora de la manipulación de los objetos los procesos o eventos continuos de fuerza física, como la abrasión, colaboren al desgaste o desprendimiento de las etiquetas que acompañan a los objetos. Dependiendo del lugar donde se encuentren, ante el posible contacto con contaminantes y plagas 
Tabla 1. Síntesis de cuadros FODA y CAME realizados como diagnóstico del Museo Regional de Rocha en el año 2012.

Table 1. Synthesis of SWOT and CAME paintings carried out as a diagnosis of the Regional Museum of Rocha in 2012.

\begin{tabular}{|c|c|}
\hline \multicolumn{2}{|c|}{ Cuadro FODA } \\
\hline Fortalezas & Oportunidades \\
\hline $\begin{array}{l}\text { - cuenta con personal estable. } \\
\text { - único museo de la ciudad y de las instituciones } \\
\text { culturales más antiguas de la localidad. } \\
\text { - ubicación estratégica. } \\
\text { - entrada gratuita. } \\
\text { - segmentos cautivos (instituciones educativas y } \\
\text { aficionados). } \\
\text { - servicio de visitas guiadas (previa coordinación). }\end{array}$ & $\begin{array}{l}\text { - Institución cultural de referencia } \\
\text { local y departamental. } \\
\text { - punto turístico y cercanía al } \\
\text { movimiento durante verano. } \\
\text { - acervo extenso y diverso. } \\
\text { - cuenta con colecciones únicas a } \\
\text { nivel nacional. } \\
\text { - edificio de } 500 \mathrm{~m} 2 \text {, con dos salas } \\
\text { permanentes y una para exposiciones } \\
\text { temporales. } \\
\text { - Proyecto Parque Natural de la } \\
\text { Cuenca de la Laguna de Rocha. }\end{array}$ \\
\hline Debilidades & Amenazas \\
\hline $\begin{array}{l}\text { - dependencia de organismo público estatal. } \\
\text { - ausencia de plan director. } \\
\text { - no cuenta con áreas de trabajo diferenciadas ni } \\
\text { roles específicos para el personal. } \\
\text { - carencia de inventario de las colecciones. } \\
\text { - carencias comunicacionales y falta de señalética } \\
\text { (urbana). } \\
\text { - problemas edilicios y de accesibilidad. }\end{array}$ & $\begin{array}{l}\text { - ausencia de políticas culturales por } \\
\text { parte de la gestión municipal. } \\
\text { - competencia por recursos } \\
\text { económicos. } \\
\text { - carencias de personal y de } \\
\text { formación. } \\
\text { - ausencia de plan educativo. } \\
\text { - percepción de museo "clásico" como } \\
\text { lugar poco atractivo. }\end{array}$ \\
\hline \multicolumn{2}{|c|}{ Cuadro CAME } \\
\hline Corregir debilidades & Afrontar amenazas \\
\hline $\begin{array}{l}\text { - diseño de plan director. } \\
\text { - rediseño guion museográfico. } \\
\text { - elaboración de protocolos de gestión de } \\
\text { colecciones. } \\
\text { - diseñar estrategias comunicacionales y de } \\
\text { visibilización del museo. }\end{array}$ & $\begin{array}{l}\text { - estrategias para generación de } \\
\text { recursos propios del museo. } \\
\text { - estrategias de promoción del } \\
\text { museo. } \\
\text { - incorporar el guion la historia local. } \\
\text { - generar propuestas educativas de } \\
\text { carácter no formal. } \\
\text { - captación de nuevos públicos. }\end{array}$ \\
\hline Mantener fortalezas & Explotar oportunidades \\
\hline $\begin{array}{l}\text { - centro de memoria local. } \\
\text { - accesibilidad de la entrada. } \\
\text { - aprovechar el enclave del museo para el diseño de } \\
\text { circuitos culturales. } \\
\text { - mantener e incrementar el acervo, promoviendo la } \\
\text { recepción de colecciones (junto con aplicación de } \\
\text { protocolo de gestión de colecciones) }\end{array}$ & $\begin{array}{l}\text { - transformarse en un centro de } \\
\text { participación ciudadana. } \\
\text { - constituirse en un museo de } \\
\text { proyección local y regional } \\
\text { (asociación con otros municipios). } \\
\text { - alternativa para el turismo de sol y } \\
\text { playa. } \\
\text { - potenciar la participación de la } \\
\text { institución con proyectos y programas } \\
\text { culturales. } \\
\text { - incorporar investigación y } \\
\text { conservación a sus funciones (a } \\
\text { través de acuerdos y convenios). }\end{array}$ \\
\hline
\end{tabular}

las etiquetas pueden sufrir degradación, o los niveles incorrectos de humedad relativa, pueden afectar a los adhesivos utilizados para fijarlas a los objetos (Waller y Cato 2009). Los efectos de la disociación pueden comprometer o incluso llevar a la pérdida de los objetos, de las colecciones y de la información que le otorga valor, a través de su contexto y significado (Waller y Cato 2009). Esta problemàtica puede surgir desde el origen, por 
ejemplo, a veces al momento de la llegada de las piezas se carece de información, no se cuenta con el inventario original o no se cuenta con uno propio.

Sobre los aspectos de conservación, si bien desde hace algunos años se está trabajando en revertir hábitos y condiciones desfavorables para los materiales en los museos en nuestro país, aún queda mucho camino por andar (Vienni y Blasco 2014). En el caso del MRR identificamos distintos agentes de deterioro que están alterando al material arqueológico en el depósito y la sala de exposición, generando un riesgo de disociación muy alto por falta de inventarios (Lamas et al. 2012) (Figuras 3 y 4). En el caso concreto del MRR, el panorama para la conservación es preocupante, con altos riesgos de humedad, agentes físicos sobre los materiales, así como de disociación (Tabla 2).

\section{Reflexiones y Discusión}

La propuesta de diagnóstico del MRR surgió en primer lugar por la necesidad de investigar colecciones vinculadas al área dónde se estaban realizando las investigaciones arqueológicas. Luego, en el contexto de nuevas acciones de extensión y reflexiones en torno de la socialización del patrimonio y la gestión de colecciones en el marco del Programa de Educación y Ciencia Pública, se plantea la necesidad de hacer una revisión y proponer otras miradas al tema.

En primer lugar, como nosotros, otros investigadores deben consultar colecciones para la colecta de datos de base como antecedentes para sus proyectos. Es aquí un tema de interés y preocupación entonces el no poseer información de origen de las colecciones debido a la disociación y/o mala conservación. Es el caso concreto del Paisaje Protegido Laguna de Rocha, donde a partir de las entrevistas realizadas se confirmó el dato de la donación de materiales del área al museo; sin embargo, hasta el momento no los hemos podido identificar en el acervo de la institución. Si bien bajo el nuevo panorama que ofrece la Ley de Museos, la documentación, protección y conservación de los bienes patrimoniales cobran un lugar significativo, es importante destacar que ésta es una tarea ardua que requiere personal destinado a ello y que se dificulta aún más cuando no se tiene información de origen de ningún tipo.

Por otra parte, parece de suma importancia en la actualidad dar respuesta al constante y legítimo reclamo de distintos sectores de la sociedad de que los materiales vuelvan a su lugar de origen. Ante esto, la arqueología demanda museos locales con condiciones propicias para alojar materiales provenientes de investigaciones científicas que en general hasta el momento no están dadas. En el Departamento de Rocha particularmente, esta situación se hace especialmente evidente: se trata de uno de los departamentos de nuestro país con mayor cantidad de investigaciones arqueológicas desde la década de 1970 y sin embargo, este acumulado de investigaciones no se traduce en propuestas museográficas que transmitan o socialicen la información a nivel local.

Generalmente tanto el resguardo como la musealización de los materiales provenientes de las investigaciones arqueológicas de Rocha se desarrollan en instituciones de Montevideo (p.e. Museo Nacional de Antropología) o permanecen en depósitos -generalmente también en lugares con condiciones de conservación deficitarias- en los laboratorios de investigación. Esta situación -que no es ajena a las de otras localidades de nuestro territorio (ver Vienni y Blasco 2014)- obstruye una mayor apropiación social del patrimonio arqueológico. Si ese patrimonio no se cuida, conserva e investiga, tampoco se puede dar a conocer ni comunicar la importancia de su salvaguarda, redundando en un desinterés de la sociedad y derivando en el consecuente abandono y exposición al deterioro (Hernández Hernández 2010). Esta situación se asemeja

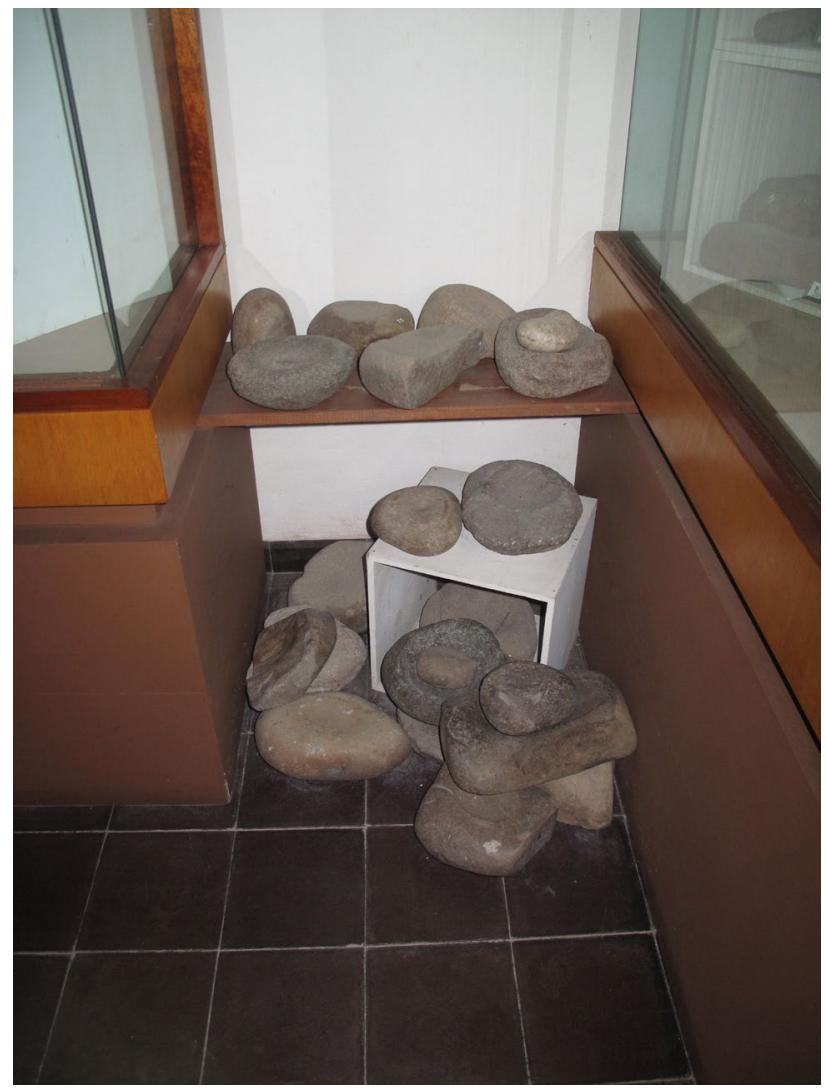

Figura 3. Situación de la exposición de materiales de molienda en MRR. El apilamiento de materiales de molienda genera daños por fricción, así como la mezcla de materiales procedentes de varias regiones de Rocha y sin leyenda. Año 2012. Fuente: Archivo LAPPU.

Figure 3. Situation of the exhibition of grinding materials in $M R R$. The stacking of general grinding materials, friction damage and mixing of material from various regions of Rocha and without legend. Year 2012. Origin: LAAPU Archive. 
G. Lamas et al.| Revista del Museo de Antropología 12 (3): 23-34 | 2019

http://dx.doi.org/10.31048/1852.4826.v12.n3.21698

Tabla 2. Estimación del riesgo de deterioro existente o potencial en el Museo Regional de Rocha (Tomado de Lamas et al. 2012:14).

Table 2. Risk estimation of existing or potential deterioration in the Regional Museum of Rocha (Taken from Lamas et al. 2012: 14).

\begin{tabular}{|c|c|c|c|}
\hline Agente de deterioro & Riesgo Bajo & Riesgo Medio & Riesgo Alto \\
\hline Humedad relativa incorrecta & & & $x$ \\
\hline Temperatura incorrecta & & $x$ & \\
\hline Radiación & $x$ & & \\
\hline Contaminantes & & $x$ & \\
\hline Fuerzas físicas directas & & & $x$ \\
\hline Robo, vandalismo & & $x$ & \\
\hline Disociación & & & $x$ \\
\hline Fuego & $x$ & & \\
\hline Agua & & $x$ & \\
\hline Plagas & & $x$ & \\
\hline
\end{tabular}

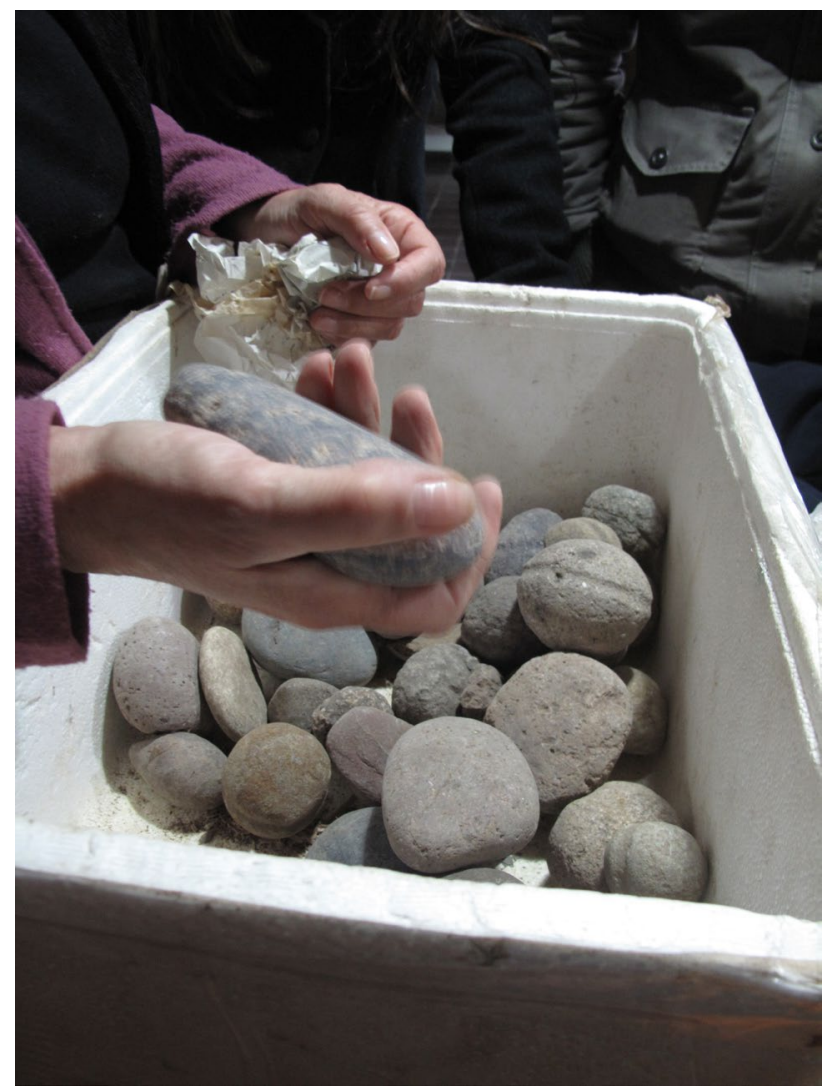

Figura 4. Fotografía de la situación de algunos materiales arqueológicos del acervo del MRR. Estos materiales fueron indicados como de procedencia "probable" de la zona de la Laguna de Rocha. Fuente: Archivo LAPPU.

Figure 4. Photographs of the situation of some archaeological materials from the MRR acquis. The origin of these materials were indicated as "probable" from Laguna de Rocha. 
a una cinta de Moebius: no se conserva lo que no se investiga y comunica y no se comunica lo que no se estudia y conserva.

Además de la conservación e investigación, las propuestas museográficas y la educación patrimonial cobran un rol importante en esta dinámica. Las actividades de este tipo orientadas a la arqueología son escasas a nivel nacional y tanto más a nivel local (Vienni y Blasco 2014), al tiempo que generalmente no hay actualización de los contenidos de las vitrinas -que en el mejor de los casos presentan datos científicos de décadas atrás-, transmitiendo conceptos generados bajo marcos teóricos en desuso. Las pocas actividades de educación puestas en relación con estos contenidos pueden entonces también verse limitadas. Si bien se depende constantemente de voluntades políticas locales, esta situación lentamente se va revirtiendo gracias al empuje de la formación de profesionales en museología y áreas afines así como a la creación del Proyecto Sistema Nacional de Museos y la legislación nacional encargada de regular el desarrollo de estas instituciones -por ejemplo el caso del Museo Arqueológico de Río Negro - MUARN y del Museo Maeso del Departamento de Soriano, ambos inaugurados en el año $2017^{6}$.

El caso de la creación MUARN es una experiencia que muestra un proceso exitoso dónde se vinculan el trabajo técnico del equipo a nivel de investigación, la vinculación con las autoridades municipales y la comunidad local desde 2009. Los técnicos y profesionales, partieron de una precaria situación registrada de las colecciones, y buscaron como estrategia para gestionar estos bienes culturales la participación de la comunidad local. Entre estas se destacan visitas a sitios arqueológicos, talleres vinculados a la temática arqueológica y el ordenamiento y acondicionamiento primario de la colección con la participación de alumnos y docentes del liceo de Young (Bortolotto et al. 2010). Como parte del proceso se realizó un inventario de cajas, con el fin de evaluar el estado relación a la colección original, y como paso fundamental para la puesta en valor y conservación preventiva (ICOM 2008) (Gascue et al. 2015). Estas instancias previas de investigación, registro y acondicionamiento de las colecciones fueron acompañadas de la rehabilitación edilicia del espacio del museo, donde también fue diseñada una propuesta museográfica con apoyaturas y cartelería vinculadas a un nuevo guión diseñado a partir del acervo del museo y por último un acondicionamiento final e inventariado del mismo (Gascue et al. 2015). Este tipo de iniciativas que se realizan de forma más cercana a los sitios contribuyen a su vez al involucramiento de la comunidad local y a la permanencia de las colecciones en las localidades. A diferencia del MUARN, el Museo Regional de Rocha no fue creado con la finalidad de ser un museo arqueológico y con la posibilidad de crear con

\footnotetext{
${ }^{6}$ Se puede acceder a la información de ambos museos en la web museos.uy
}

concepciones actualizadas toda la museografía y gestión del acervo, por el contrario el mismo es una referencia a nivel local por lo que los planteos de reorganización, modificaciones o para realizar los diferentes cambios son necesarios mayores consensos tanto con la comunidad local como con los funcionarios y las autoridades municipales.

Por otro lado, entendemos que la oferta esencial del museo son las exposiciones, a través de las cuales se pone en práctica una actividad que se torna un instrumento al servicio de la difusión de los contenidos del museo y de sus colecciones, y para difundir a la institución misma (Valdés Sagüés 2008). Pero además, los museos a través de su museografía y de las fórmulas de comunicación que le son específicas, son también instrumentos al servicio del patrimonio cultural que pueden aportar, de forma sencilla y entretenida, a su conocimiento, valoración, conservación, difusión y disfrute (Hernández Hernández 2010). Particularmente, los museos arqueológicos permiten poner a disposición de todos los ciudadanos las interpretaciones sobre el pasado (especialmente las científicas), las cuales sean o no motivo de disputa, permiten a la sociedad en su conjunto entender mejor el presente y posicionarse sobre él para poder proyectarse a futuro. Sin embargo, la falta de actualización conceptual aunada al desconocimiento acerca del origen y características de los elementos que componen los acervos, conduce a generar o mantener ciertos discursos identitarios (sensu Prats 2006) que perpetúan una mirada ajena sobre el patrimonio, con concepciones lineales y evolutivas del pasado (Cabrera Pérez 2011) y con una distancia entre el presente y el pasado indígena que ha silenciado de forma sistemática la diversidad cultural original de nuestro territorio reproduciendo discursos colonialistas y patriarcales (López Mazz 2004). En este sentido, generalmente -y sin mayor desarrollo de marco interpretativo-, las exposiciones se presentan como una acumulación de piezas arqueológicas que comparten ciertas características en común y no bajo un discurso articulado (Pupio 2007). Los criterios de interpretación utilizados, bajo los lineamientos imperantes del evolucionismo lineal (Cabrera Pérez 2011) -y en menor medida del difusionismo-implican criterios clasificatorios basados en escalas de valores subjetivas con referencias en la cultura occidental y el concepto de "civilización" (Malán 2013).

\section{CONSIDERACIONES FINALES}

En base al diagnóstico específico en el Museo Regional de Rocha, se discutieron algunas líneas de acción a desarrollar de cara a mejorar aspectos del museo y específicamente concernientes al manejo de colecciones arqueológicas. Para realizar el diagnóstico se partió de situaciones ideales en base a las cuales se discutió el desarrollo de acciones de conservación preventiva en función de la realidad y necesidades de la institución 
y de su acervo. Las propuestas de acción resultantes estuvieron orientadas en tres direcciones: la prevención de daños físicos; la prevención de pérdida de información y la sensibilización tanto de público general como de autoridades municipales y personal del museo.

Si bien entendemos qué como equipo de investigación externo, ya que ninguno de los autores pertenecemos al museo, no se cuenta con la capacidad ni responsabilidad de generar cambios dentro de las instituciones, creemos que como profesionales nos cabe un rol de contribuir, aportar, sugerir cambios o formas de ordenar, clasificar, conservar, etc. A su vez, fuera de la institución, y en el marco de las investigaciones y actividades de extensión en la región, llevamos adelante actividades de educación patrimonial y socialización que sensibilicen y promuevan una mayor apropiación del patrimonio arqueológico.

En el caso concreto, se analiza un caso, el del MRR, pero existen diversas iniciativas de museos departamentales y locales que deben y pueden también ser analizados en estos términos. La discusión sobre la creación por ejemplo de Centros de Interpretación para las áreas protegidas del SNAP es una oportunidad para proyectar el albergue de las colecciones locales, así como para la construcción de nuevos marcos interpretativos para el patrimonio arqueológico no lineales que se basen en la multiculturalidad, decolonizadoras y que no sean globalizantes. En este sentido, la generación de narrativas que involucren acciones participativas o que desarrollen miradas no eurocéntricas y en donde se parta de la idea de no dualidad de la historia, la evolución y/o de la separación entre naturaleza y sociedad (Quijano 2000).

Dado que en el MRR la mayoría de los objetos y colecciones que recibe el museo provienen de donaciones realizadas por particulares o instituciones sin inventarios asociados, es necesario seguir desarrollando el trabajo sobre las colecciones arqueológicas en custodia de particulares. Para que el destino ideal futuro de esas colecciones sea el museo local, este doble abordaje es importante e hilvana el vínculo entre el particular y la institución pública que garantiza su cuidado y disfrute. Para esto es necesario que se retome la confianza en este tipo de instituciones tanto con los particulares como con los investigadores.

Desde la arqueología, este caso de análisis concreto, por un lado nos interpela como voz legitimadora de interpretaciones y narrativas acerca del pasado y del patrimonio local; por otro deja entrever la necesidad de fortalecer el ejercicio reflexivo, de análisis y de acción que apueste al conocimiento, cuidado y disfrute del patrimonio arqueológico por parte de toda la ciudadanía y a un diálogo abierto que ponga su mirada sobre lo museos como instituciones capaces de promover visiones críticas y transformaciones sociales en pos de darle lugar a los sectores invisibilizados.
Ante la pregunta de "¿quién se beneficia de la teoría y práctica y del discurso arqueológico? (Funari 2001:1) se hace vital en nuestro contexto desarrollar y fortalecer la arqueología pública, entendida como aquella que aborda, de forma crítica desde la teoría y la acción, los procesos de apropiación del pasado, así como las relaciones existentes en el presente entre la arqueología y la sociedad para lograr una mejor coexistencia entre ambas y potenciar el entendimiento sobre el valor y uso de la disciplina (Almansa Sánchez 2011; Salerno 2013). Mientras se sigan realizando intervenciones arqueológicas y generando colecciones científicas, es necesario, de forma paralela, seguir cuestionándose y reflexionando desde la propia disciplina acerca del rol que se juega en la construcción del patrimonio y en la conformación de los acervos y discursos dentro de los museos.

En contrapartida, desde la museología se deberá atender a los (no tan) nuevos retos que plantea el patrimonio frente a la memoria colectiva, la diversidad cultural, las minorías invisibilizadas y las identidades. Los museos, deberán comprometerse cada vez más en garantizar las condiciones mínimas e indispensables para que los testimonios materiales de la diversidad puedan ser conservados y socializados.

Montevideo, 26 de noviembre de 2019

\section{Agradecimientos}

Queremos agradecer muy especialmente a la ex-Directora del MRR Sra. Matilde Rodríguez por abrirnos las puertas y la colaboración brindada. A Bruno Gentile, Carla Bica, Oscar Marozzi, Camila Gianotti y a nuestros compañeros y compañeras del LAPPU quienes son parte de la investigación y con quienes compartimos y reflexionamos cotidianamente. A Elena Saccone por la colaboración en la revisión del resumen. Agradecemos los comentarios y sugerencias de los revisores anónimos de la Revista del Museo de Antropología.

\section{Bibliografía}

Almansa Sánchez. J. (2011) Arqueología para todos los públicos. Hacia una definición de la Arqueología Pública "a la española". En ArqueoWeb, 13:87-107.

Arocena, R. (2011) Curricularización de la extensión: ¿por qué, cuál, cómo?. en "Integralidad: Tensiones y Perspectivas. Cuaderno $N^{\circ} 1$ de Extensión. Cuadernos de Extensión. Comisión Sectorial de Extensión y Actividades en el Medio (CSEAM). https://www.extension.udelar.edu. uy/wp-content/uploads/2017/11/Cuaderno-n\%C2\%B01integralidad.pdf

Blasco, J., N. Bortolotto y G. Lamas (2017) Gestión de Colecciones Arqueológicas: Un desafío constante. En Cuaderno de resúmenes de ponencias y actividades 
de las Jornadas Académicas 2017 Prof. Washington Benavídez, pp. 393. FHCE, Udelar, Montevideo.

Bortolotto, N., A. Gascue, J. Baeza, J. Gómez Tincabelli, J.Lemos Zito y Ch. Duarte de Aarmas 2010. Extendiendo la Arqueología. Valorización del patrimonio cultural prehistórico en la enseñanza secundaria. Actas de las III Jornadas de Investigación y II de Extensión. Facultad de Humanidades y Ciencias de la Educación, pp. 21. http:// www.fhuce.edu.uy/jornada/2010/PONENCIAS/ BORTOLOTTOetal.PDF

Cabrera Pérez, L. (2011). Patrimonio y Arqueología en la región platense. CSIC. Udelar, Montevideo.

Curbelo, M. C (2004) Reflexiones sobre el desarrollo teórico del pensamiento en la arqueología uruguaya. En Teoría arqueológica en américa del sur, editado por G. Politis y R. Peretti, pp 259-279, serie teórica n³, ICUAPA, Argentina.

Desvallés, A.y F. Mairesse (2010) Conceptos claves de museología. ICOM.

Finola, H.A. (2016) Memorias en conflicto alrededor del Museo arqueológico Gustavo Le Paige, en San Pedro de Atacama, Chile. Nuevo Mundo Mundos Nuevos [En ligne], Questions du temps présent, mis en ligne le 25 janvier 2016, consulté le 11 novembre 2019. URL : http:// journals.openedition.org/nuevomundo/69148; DOI: 10.4000/nuevomundo.69148

Funari, P. P. (2001) Public archaeology from a Latin American perspective. En Public Archaeology, American Anthropological Association. 1, 4, 2001, pp. 239-243.

Gascue, A., N. Bortolotto y M. Fleitas. (2015). Conservación y musealización de la Colección Arqueológica GALY. En Cuadernos del Instituto Nacional de Antropología y Pensamiento Latinoamericano - Series Especiales, $N^{\circ} 2$ Vol. 3, pp. 71-86.

Gianotti, C., y E. Villarmarzo. (2011). Relevamiento arqueológico rápido, identificación de elementos culturales de conservación del Área Protegida Laguna de Rocha. Actividad 4, Tarea 4.5. In Consultoría técnica para apoyar la elaboración del plan de manejo del Paisaje Protegido Laguna de Rocha. Futuro Sos. Montevideo.

Gómez, M. y B. de Tapol 2009 Medio siglo de Conservación Preventiva. Entrevista a Gaël de Guichen. Entrevista realizada por el Comité Científico Técnico del GEIIC (Marisa Gómez y Benoît de Tapol) en agosto de 2009. En Ge Conservación, Nº, pp. 35-44.

Hernández Hernández, F. (2006). Planteamientos teóricos de la museología. Ediciones Trea, España.
Hernández Hernández, F. (2010). Los museos arqueológicos y su museografía. Ediciones Trea, España.

Lamas, G., C. Bica, J. Blasco, E. Villarmarzo, B. Vienni, C. Gianotti, O. Marozzi, y B. Gentile. (2012). Informe Diagnóstico. Proyecto Actividad de extensión CSEAM 2012. Paisajes culturales y diagnóstico participativo en Museo Regional de Rocha Milton de los Santos. Rocha, $17 \mathrm{pp}$.

Llop I Bayó, F. 1996. Los inventarios, herramienta de creación del patrimonio etnológico. Catalogación del Patrimonio Histórico. Cuadernos VI del Instituto Andaluz del Patrimonio Histórico. Junta de Andalucía. Conserjería de Cultura. Sevilla, pp. $49-51$.

López Mazz, José Ma. (2004). Arqueología e identidad uruguaya: el saber y el poder en las vanguardias intelectuales. In Teoría arqueológica en America del Sur, edited by Gustavo Politis and Roberto Peretti, pp. 197-211. Facultad d. UNICEN, Olavarría.

López Mazz, José Ma. (2008). Investigación y Propuesta de Trabajo. El Componente Cultural en el Área de Reserva de Biosfera Bañados del Este. UNESCO, Montevideo.

Malán, M. (2013) Aportes desde la Arqueología en la revalorización de patrimonios locales. Una propuesta de gestión en torno a la colección arqueológica "René Mora". Cuadernos del Instituto Nacional de Antropología y Pensamiento Latinoamericano - Series Especiales No1 (3), Argentina.

Michalsky, S. (2006) Preservación de las colecciones. En Cómo administrar un museo: Manual práctico, pp. 5190. ICOM, París.

Podgorny, I. (2005) La mirada que pasa: museos, educación pública y visualización de la evidencia científica. História, Ciências, Saúde-Manguinhos, v. 12 (suplemento), p. 231-64, 2005.

Prats, LI. (2006). La mercantilización del patrimonio: entre la economía turística y las representaciones identitarias. Revista Ph 58: 72-80.

Pupio, Alejandra. (2007). Arqueólogos y coleccionistas en la formación del Patrimonio Arqueológico en la Provincia de Buenos Aires en la década de 1950. In Arqueología en las Pampas. Tomo II, editado por Cristina Bayón, Alejandra Pupio, Ma. Isabel González, Nora Flegenheimer, and Magdalena Frere, pp. 783-798. 1a. Sociedad Argentina de Antropología, Buenos Aires.

Quijano, Aníbal. (2000). Colonialidad del poder, eurocentrismo y América Latina. In La colonialidad del saber: eurocentrismo y ciencias sociales. Perspectivas 
Latinoamericanas., editedby Edgardo Lander, pp. 201246. CLACSO. Buenos Aires.

ROU. 2010. Decreto № 61/010 (18 de febrero de 2010). Laguna de Rocha. Selección y delimitación del área natural protegida.

Salerno, V. (2013). Arqueología pública: reflexiones sobre la construcción de un objeto de estudio. En Revista Chilena de Antropología, N²7. pp. 7-38. Universidad de Chile, Santiago, Chile.

Salerno, V.M. y M. Vigna (2011). Acercamiento a la construcción del pasado prehispánico en una sala del Museo Pampeano de Chascomús entre 1939 Y 1992 Arqueología 18:181-207 • 2012 • Instituto de Arqueología • FFyL. UBA. ISSN en línea 1853-8126.

Sosa, M., J. Blasco y L. Bergatta. (2014). Conservar para Investigar: las colecciones arqueológicas del Museo de Arte Precolombino e Indígena. En Libro de resúmenes del Il Congreso Internacional de Arqueología de la Cuenca del Plata, pp. 15, Facultad de Humanidades y Ciencias de la Educación, Montevideo, Uruguay.

Tommasino, H.; Cano, A.; Castro, D.; Santos, C.; Stevenazzi, F. (2010): “De la extensión a las prácticas integrales", en H. Tommasino (coord.), Hacia la Reforma Universitaria. La extensión en la transformación de la enseñanza: los espacios de formación integral, Montevideo: Udelar.

Vienni, Bianca y Jimena Blasco. (2014). Museos y socialización del patrimonio arqueológico en Uruguay. En: Museologia e Patrimônio - Revista Eletrônica do Programa de Pós-Graduação em Museologia e Patrimônio - Unirio | MAST - vol.7, n.2, 2014.

Vienni, Bianca, Eugenia Villarmarzo, Camila Gianotti, Jimena Blasco, Carla Bica, y Gastón Lamas. (2011). Ciencia Pública en construcción: El Programa de Educación patrimonial y Ciencia Pública del LAPPU (FHCE). In IV Jornadas de Investigación y III Jornadas de Extensión de la Facultad de Humanidades y Ciencias de la Educación, octubre 2011, Montevideo. Ponencia GT35, edited by FHCE, pp. 1-12. Udelar, Montevideo.

Valdés Sagües, M. C. (2008). La difusión, una función del museo. En: Museos.es: Revista de la Subdirección General de Museos Estatales, No 4, pp.64-75. Disponible en: http://www.mcu.es/museos/docs/MC/MES/Rev04/ Desde_Difusion_funcion_museoC_Valdes.pdf (Acceso: 20/07/2012).

Villarmarzo, E. 2017. Las lagunas costeras y su rol en la ocupación de las Tierras Bajas: El caso de Laguna de Rocha. Cuadernos do Lepparq (En prensa).

Villarmarzo, E., J. Blasco, G. Lamas, B. Gentile. (2017). Nuevos aportes a la Gestión integral del Paisaje Protegido Laguna de Rocha: investigación y sensibilización en torno a colecciones arqueológicas. VII Jornadas de Investigación, VI Jornadas de Extensión, V Encuentro de Egresados y Estudiantes de Posgrado de la Facultad de Humanidades y Ciencias de la Educación, 11-13 octubre de 2017, Montevideo. (EP)

Waller R. y P. S. Cato. (2009). Disociación. ICCROM (2009) (edición en español).

\section{Documentos electrónicos}

Ley de Museos y Sistema Nacional de Museos № 19.037. Disponible en: http://archivo.presidencia.gub.uy/sci/ leyes/2012/12/mec_837.pdf

ICOM (2008) Terminología para definir la conservación del patrimonio cultural tangible. Disponible en: http:// www.icom-cc.org/54/document/icom-cc-resolucionterminologia-espanol/?action=Site_Downloads_ Downloadfilefid=748). 